\title{
Clinical Usefulness of Loudness Dependence of Auditory Evoked Potentials (LDAEP) in Patients with Bipolar Disorder
}

\author{
Young-Min Park ${ }^{1}$ and Seung-Hwan Lee ${ }^{1,2} \bowtie$ \\ ${ }^{1}$ Department of Psychiatry, Ilsan Paik Hospital, Inje University College of Medicine, Goyang, Republic of Korea \\ ${ }^{2}$ Clinical Emotion and Cognition Research Laboratory, Goyang, Republic of Korea
}

While it has been reported previously that the loudness dependence of auditory evoked potentials (LDAEP) is a putative biological marker or a predictor of treatment response, there have been few studies of LDAEP in bipolar disorder. However, a recent study by Park and colleagues raised the possibility that the LDAEP could be useful as a biological marker of bipolar disorder. They found that the LDAEP was significantly higher in normal controls than in patients with either bipolar disorder or schizophrenia. Lee and colleagues also examined the LDAEP in bipolar disorder and normal controls, and found that it differed according to the bipolar phase, being significantly higher in cases of euthymic bipolar disorder, bipolar depression, and bipolar mania. With regard to treatment response, early clinical findings were that a higher LDAEP and a stronger intensity dependence of visual evoked potentials were related to a favorable response to lithium treatment. Juckel and colleagues recently demonstrated that the pretreatment LDAEP could be a predictor of successful prophylactic lithium treatment. The present article reviews the literature in order to determine whether the LDAEP can be used as a biological marker or a predictor of treatment response in patients with bipolar disorder and of manic switch or treatment resistance in patients with major depressive episode(s).

Psychiatry Investig 2013;10:233-237

Key Words LDAEP, Bipolar disorder, Bipolarity, Treatment response, Biological marker, Manic switch, Treatment resistance.

\section{INTRODUCTION}

The pathophysiology related to serotonin in bipolar disorder remains unclear. Some investigators have assumed that a serotonin deficit is associated with the onset of bipolar mania, and that an increase in serotonin activity induces a mood-stabilizing action. ${ }^{1}$ In contrast, it has also been reported that tryptophan depletion decreases manic symptomatology. ${ }^{2}$ Fountoulakis and colleagues recently suggested that bipolar depression is related to both serotonin and norepinephrine activity. ${ }^{3}$ In addition, it was reported that the biosynthetic enzymes for both norepinephrine and serotonin are reduced in the locus ceruleus in patients with bipolar depression who commit suicide, but not in those with unipolar depression who commit suicide. ${ }^{4}$ Likewise, a blunted prolactin response to fenflura-

Received: August 5, 2013 Revised: August 6, 2013

Accepted: August 6, 2013 Available online: September 16, 2013

$\triangle$ Correspondence: Seung-Hwan Lee, MD, $\mathrm{PhD}$

Department of Psychiatry, Ilsan Paik Hospital, Inje University College of Medicine, 170 Juhwa-ro, Ilsanseo-gu, Goyang 411-706, Republic of Korea Tel: +82-31-910-7260, Fax: +82-31-910-7268, E-mail: 1shpss@hanmail.net

(a) This is an Open Access article distributed under the terms of the Creative Commons Attribution Non-Commercial License (http://creativecommons.org/licenses/bync/3.0) which permits unrestricted non-commercial use, distribution, and reproduction in any medium, provided the original work is properly cited. mine administration revealed that central serotonergic activity in patients with bipolar depression was impaired. ${ }^{5}$

It was recently suggested that the loudness dependence of auditory evoked potentials (LDAEP) is a valid marker of the central serotonergic activity. It has been demonstrated that the LDAEP is inversely associated with central serotonergic activity, with a high LDAEP reflecting low levels of serotonergic neurotransmission. ${ }^{6,7}$ The LDAEP is reportedly a putative biological marker. Gudlowski and colleagues found that the LDAEP was significantly lower in schizophrenia patients than in normal controls, ${ }^{8}$ whereas Linka and colleagues found that the LDAEP did not differ significantly between patients with major depressive disorder (MDD) and normal controls. ${ }^{9}$ Yet other investigators found a difference in the LDAEP between patients with melancholic depression and those with nonmelancholic depression. ${ }^{10}$

Until recently there have been few studies examining the LDAEP status in bipolar disorder. However, in 2010, Park et al. ${ }^{11}$ reported that as in schizophrenia, the LDAEP is significantly lower in bipolar patients than in normal controls and patients with MDD. In another study, Lee et al. ${ }^{12}$ investigated the LDAEP of multiple mood statuses (bipolar depression, bipolar mania, and euthymia) and assessed its clinical usefulness 
in patients with bipolar disorder. Furthermore, Park and Lee $\mathrm{L}^{13}$ examined the LDAEP in patients with MDD who presented with subthreshold bipolarity.

The aim of the present study was to review the literature with a view to determining whether the LDAEP can be used as a biological marker or a predictor of treatment response in patients with bipolar disorder and of manic switch or treatment resistance in patients with major depressive episode(s).

\section{LDAEP AS A BIOLOGICAL MARKER IN BIPOLAR DISORDER}

Some studies have shown that the LDAEP is a putative biological marker in several psychiatric disorders. ${ }^{8,11,14}$ Gudlowski et al. ${ }^{8}$ explored the LDAEP in 60 patients with prodromal symptoms of schizophrenia, 34 patients with first-episode of schizophrenia, 28 patients with a chronic course of schizophrenia, and 57 normal controls. They found that in the schizophrenia patients the LDAEP was already low before the onset of the disorder, and remained decreased throughout the disease progression. Thus, a low LDAEP may be a trait marker rather than a state marker, and may correspond to the serotonin hypothesis of schizophrenia. In 2010, Park et al. ${ }^{11}$ suggested for the first time the possibility that the LDAEP could be used as a biological marker in bipolar disorders. Their subjects comprised 55 normal controls, 123 patients with MDD, 37 with bipolar disorder, 46 with schizophrenia, 37 with panic disorder, 31 with generalized anxiety disorder (GAD), and 28 with post-traumatic stress disorder. They found that LDAEP was significantly higher in normal controls than in patients with either bipolar disorder or schizophrenia, and did not differ significantly between normal controls and patients with MDD or GAD. Thus, there appears to be a higher level of central serotonergic activity in bipolar disorder and schizophrenia, and MDD might be a heterogeneous entity. In particular, the results of Park and colleagues related to schizophrenia are consistent with those of Gudlowski et al. ${ }^{8}$ However, these studies were subject to some limitations. For example, LDAEP was measured during a time when the subjects had been medicated, and these medications, such as antipsychotics and mood stabilizers, may have affected the LDAEP. In addition, the phases of the bipolar patients were not controlled; that is, the mean LDAEP in bipolar disorder was calculated using the LDAEP in all patients with bipolar mania, bipolar depression, and euthymic bipolar disorder. That being said, the study of Park et al. is the first to reveal the potential of LDAEP as a trait marker in bipolar disorder.

Lee et al. ${ }^{12}$ also examined the LDAEP in patients with bipolar disorder and in normal controls. Their subjects comprised 35 patients with bipolar disorder, 32 patients with schizophre- nia, and 22 healthy controls; the patients with bipolar disorder comprised 10 with bipolar depression, 15 with bipolar mania, and 10 with euthymic bipolar disorder. They found that the LDAEP differed according to the bipolar phase, and was significantly higher in cases of euthymic bipolar disorder, bipolar depression, and bipolar mania. The LDAEP was significantly higher in patients with euthymic bipolar disorder than in patients with schizophrenia, higher in bipolar depression than in schizophrenia, higher in normal controls than in patients with schizophrenia, and higher in normal controls than in patients with bipolar mania (Figure 1). These results indicate that the serotonergic activity in patients with bipolar disorder may vary according to their mood phase. The main limitation of that study was that the LDAEP was measured when the patients were under medication for their condition with drugs such as antipsychotics, antidepressants, and mood stabilizers.

Park and Lee ${ }^{13}$ examined the LDAEP in MDD with and without bipolarity. In total, 61 patients who met the criteria for the Diagnostic and Statistical Manual of Mental Disorders, edition IV, text revision, and who had no history of hypomanic or manic episodes were enrolled. The patients were divided into two subgroups based on whether or not they achieved a positive score for the Korean versions of the Mood Disorder Questionnaire. The LDAEP was measured before beginning

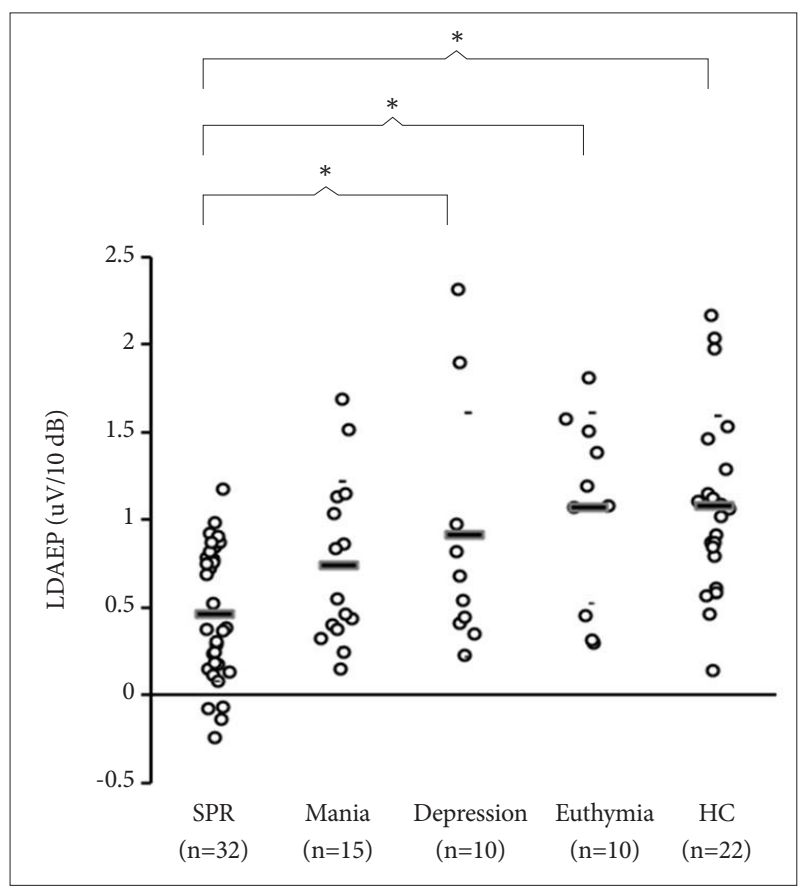

Figure 1. Comparison of loudness dependence of the auditory evoked potential (LDAEP) among schizophrenia (SPR), bipolar mania (Mania), bipolar depression (Depression), bipolar euthymia (Euthymia), and healthy controls (HC). Mean values were presented as horizontal bars. There was a strong trend of significant effect of the diagnostic groups. *represents a statistically significant difference between 2 groups $(p<0.05)$ (reprinted from Lee et al. ${ }^{12}$ with permission). 
medication with serotonergic agents. Park and Lee found that the LDAEP did not differ significantly between MDD patients with and without bipolarity (Figure 2). Although their findings were negative, their study was the first to examine LDAEP in patients with bipolarity. The findings suggest that the serotonergic activity does not differ between MDD patients with and without bipolarity.

\section{LDAEP AS A PREDICTOR OF TREATMENT RESPONSE IN BIPOLAR DISORDER}

There is growing evidence that the LDAEP is a predictor of the treatment response to antidepressants or lithium in affective disorders or GAD. ${ }^{15-17}$ Some investigators found that a high pretreatment LDAEP is associated with a favorable response to acute selective serotonin reuptake inhibitor (SSRI) treatment in patients with MDD. ${ }^{15}$ In contrast, patients with MDD who responded to reboxetine, a noradrenergic antidepressant, were reportedly characterized by a low pretreatment LDAEP. ${ }^{15}$

Park and colleagues found that it is possible for LDAEP to predict the treatment response to escitalopram in patients with GAD. ${ }^{16}$ Using a patient cohort of 25 , they found that a higher pretreatment LDAEP was related to a favorable response to acute escitalopram treatment. These results are line with those obtained in other studies involving patients with MDD. Thus, serotonergic agents such as SSRIs can produce a better treatment outcome in patients with MDD or GAD with deficits in central serotonergic activity. ${ }^{15,16}$

In the case of bipolar disorder, the early clinical findings

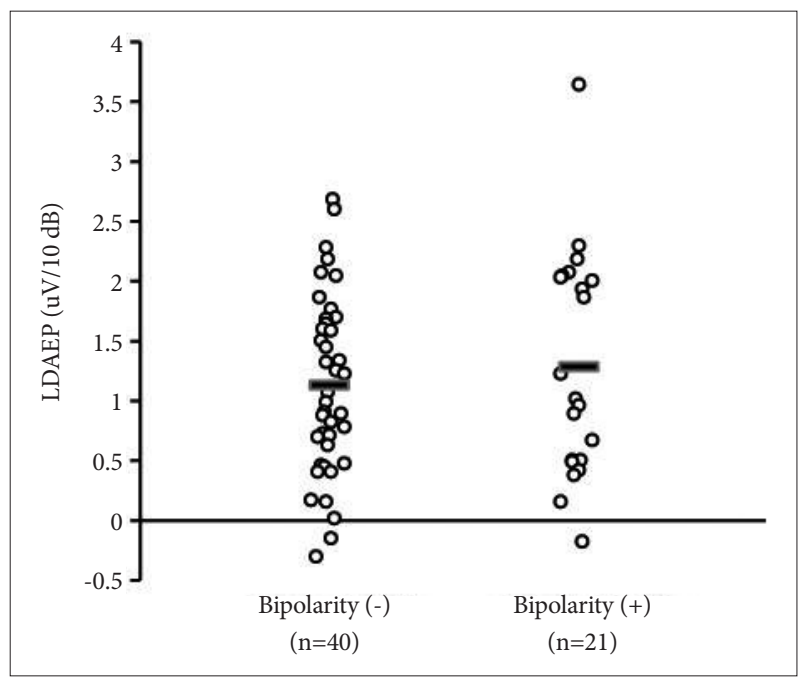

Figure 2. Comparison of LDAEP between depressive patients with bipolarity and depressive patients without bipolarity. Mean values were presented as horizontal bars. There was no significant difference between two groups (reprinted from Park and Lee ${ }^{13}$ with permission). LDAEP: loudness dependence of auditory evoked potentials. were that a higher LDAEP and a stronger intensity dependence of visual evoked potentials were related to a favorable response to lithium treatment. ${ }^{18-20}$ In addition, Juckel and colleagues found that the pretreatment LDAEP could be a predictor of successful prophylactic lithium treatment. ${ }^{17}$ Thirty patients with unipolar and bipolar affective disorder were enrolled in their study. The pretreatment LDAEP was significantly higher in the patients who responded to lithium treatment than in the nonresponders. These findings indicate that a lower pretreatment serotonergic activity was associated with a better treatment response to lithium.

Furthermore, a high LDAEP is now also considered a potential predictor of a positive lithium response in affective psychosis. ${ }^{21,22}$ One of these studies measured the LDAEP in 28 patients with euthymic affective psychosis ${ }^{22}$ who had all been treated with lithium for at least 5 years. These subjects were divided into two subgroups (responders and nonresponders) based on whether or not they had been hospitalized due to relapses during the previous 5 years of lithium therapy. The findings revealed that the LDAEP was higher in responders than in nonresponders. Another study enrolled 34 stabilized patients with affective psychosis who had been treated with lithium for at least 3 years, ${ }^{21}$ and also found that the LDAEP was higher in responders than nonresponders. Those authors hypothesized that patients with low serotonergic activity who respond to serotonin enhancers such as lithium are characterized by a high LDAEP.

\section{A HYPOTHESIS: THE LDAEP PREDICTS A MANIC SWITCH OR TREATMENT RESISTANCE IN MOOD DISORDER}

In a clinical study, Lee and colleagues observed that the LDAEP of 166 patients with MDD ranged between 0.21 and

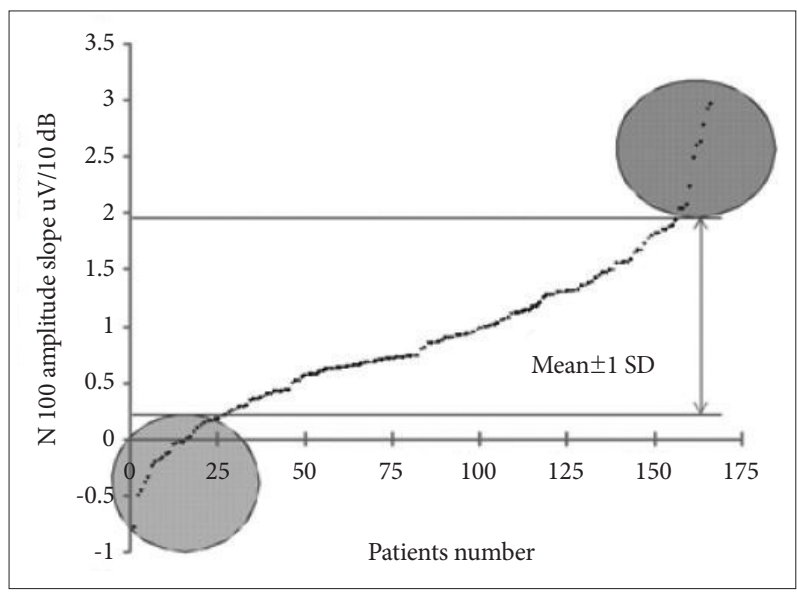

Figure 3. Distribution of the $\mathrm{N} 100$ amplitude slope in 166 patients with major depression (38 males and 128 females) and aged $47.6 \pm$ 18.4 years (mean $\pm S D$ ) (reprinted from Lee et al. ${ }^{23}$ with permission). 


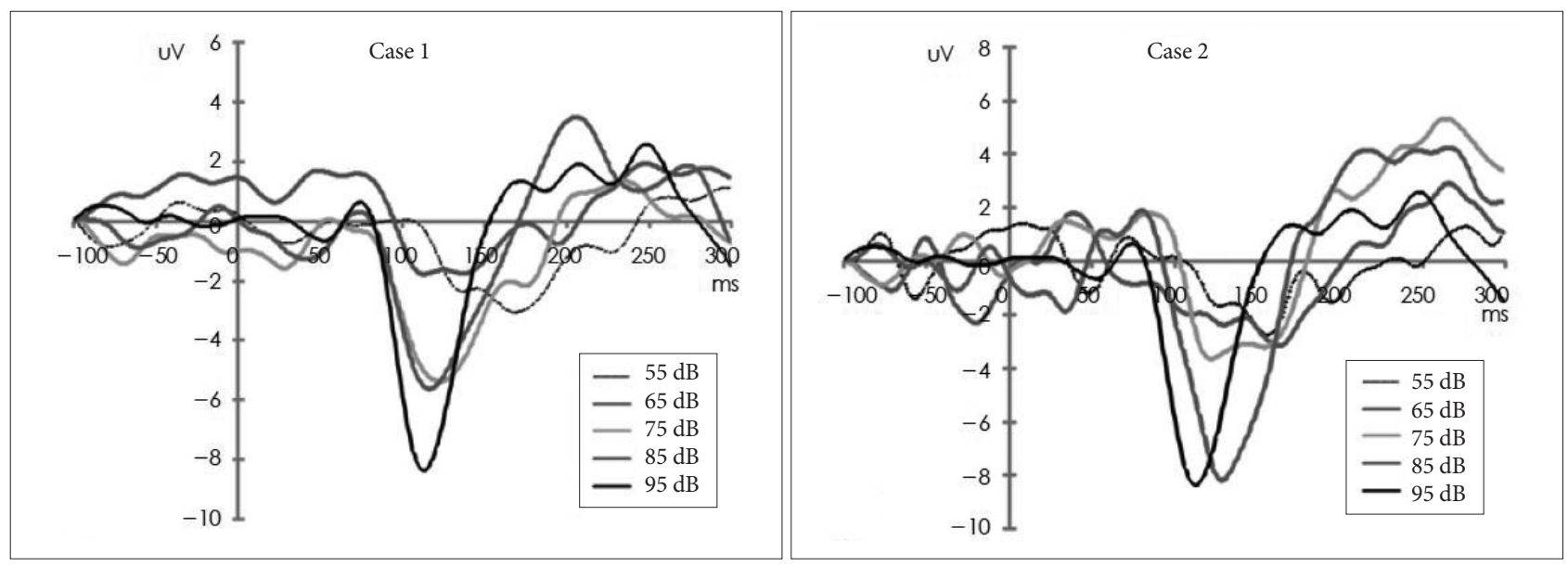

Figure 4. Illustrations of LDAEP values at the $\mathrm{Cz}$ electrode (reprinted from Lee et al ${ }^{23}$ with permission). LDAEP: loudness dependence of auditory evoked potentials.

$1.59 \mu \mathrm{V} / 10 \mathrm{~dB}$ (Figure 3). ${ }^{23}$ In summary, it can be assumed that patients with LDAEP values outside this range (which is indicated by the gray regions in the figure) would have an aberrant response to SSRIs, reflecting manic switch or treatment resistance. There is some evidence that the response of patients with MDD or GAD to SSRIs treatment improves as the LDAEP increases. ${ }^{15,16,24}$ Thus, if the LDAEP is very high, it could induce a manic switch. Conversely, there is also evidence that a low LDAEP is indicative of a worse treatment response to SSRIs, ${ }^{15}$ such that a very low LDAEP could induce treatment resistance.

Intriguingly, Lee and colleagues reported on two patients with MDD who presented with manic switch after receiving SSRI treatment. The patients had aberrantly high LDAEP values of 2.69 and 3.82 (Figure 4). It can be assumed that persistent severely low serotonergic activity (as marked by an aberrantly high LDAEP) would render the serotonin receptors supersensitive and up-regulated. The application of SSRI treatment in such a state could induce an explosive enhancement of central serotonergic action, causing a manic switch. Thus, an aberrantly high LDAEP may be a biological marker for predicting manic switch after medication of MDD patients with SSRIs or serotonergic agents.

In contrast, Park et al. ${ }^{25}$ reported on a patient with MDD who experienced severe adverse effects after taking SSRIs without improvement of his depressive symptoms. These adverse effects disappeared and his depressive symptoms improved after discontinuation of the SSRIs and replacement with tianeptine. The patient's LDAEP was very low $(-0.14)$ at baseline, which means that his central serotonergic activity was already high. It was hypothesized that the high serotonergic activity had rendered him unresponsive to SSRIs and brought on the adverse effects, and that the tianeptine was effective due to its lack of serotonin-related action. Thus, LDAEP may be used to predict the clinical response to SSRIs in patients with MDD. More studies with larger samples are needed to examine further the potential of LDAEP as a biological marker or predictor of treatment response.

\section{Acknowledgments}

This study was supported by a grant from National Research Foundation of Korea (NRF), funded by Ministry of Education and Science Technology (MEST) (2011-0010562).

\section{REFERENCES}

1. Shiah IS, Yatham LN. Serotonin in mania and in the mechanism of action of mood stabilizers: a review of clinical studies. Bipolar Disord 2000;2:77-92.

2. Applebaum J, Bersudsky Y, Klein E. Rapid tryptophan depletion as a treatment for acute mania: a double-blind, pilot-controlled study. Bipolar Disord 2007;9:884-887.

3. Fountoulakis KN, Kelsoe JR, Akiskal H. Receptor targets for antidepressant therapy in bipolar disorder: an overview. J Affect Disord 2012; 138:222-238.

4. Wiste AK, Arango V, Ellis SP, Mann JJ, Underwood MD. Norepinephrine and serotonin imbalance in the locus coeruleus in bipolar disorder. Bipolar Disord 2008;10:349-359.

5. Sher L, Oquendo MA, Li S, Ellis S, Brodsky BS, Malone KM, et al. Prolactin response to fenfluramine administration in patients with unipolar and bipolar depression and healthy controls. Psychoneuroendocrinology 2003;28:559-573.

6. Hegerl U, Juckel G. Intensity dependence of auditory evoked potentials as an indicator of central serotonergic neurotransmission: a new hypothesis. Biol Psychiatry 1993;33:173-187.

7. Hegerl U, Gallinat J, Juckel G. Event-related potentials. Do they reflect central serotonergic neurotransmission and do they predict clinical response to serotonin agonists? J Affect Disord 2001;62:93-100.

8. Gudlowski Y, Ozgurdal S, Witthaus H, Gallinat J, Hauser M, Winter C, et al. Serotonergic dysfunction in the prodromal, first-episode and chronic course of schizophrenia as assessed by the loudness dependence of auditory evoked activity. Schizophr Res 2009;109:141-147.

9. Linka T, Sartory G, Bender S, Gastpar M, Muller BW. The intensity dependence of auditory ERP components in unmedicated patients with major depression and healthy controls. An analysis of group differences. J Affect Disord 2007;103:139-145.

10. Fitzgerald PB, Mellow TB, Hoy KE, Segrave R, Cooper NR, Upton DJ, 
et al. A study of intensity dependence of the auditory evoked potential (IDAEP) in medicated melancholic and non-melancholic depression. J Affect Disord 2009;117:212-216.

11. Park YM, Lee SH, Kim S, Bae SM. The loudness dependence of the auditory evoked potential (LDAEP) in schizophrenia, bipolar disorder, major depressive disorder, anxiety disorder, and healthy controls. Prog Neuropsychopharmacol Biol Psychiatry 2010;34:313-316.

12. Lee KS, Park YM, Lee SH. Serotonergic dysfunction in patients with bipolar disorder assessed by the loudness dependence of the auditory evoked potential. Psychiatry Investig 2012;9:298-306.

13. Park YM, Lee SH. Can the loudness dependence of auditory evoked potentials and suicidality be used to differentiate between depressive patients with and without bipolarity. Psychiatry Investig 2013;10:143147.

14. Kim DH, Park YM. The association between suicidality and serotonergic dysfunction in depressed patients. J Affect Disord 2013;148:72-76.

15. Juckel G, Pogarell O, Augustin H, Mulert C, Muller-Siecheneder F, Frodl T, et al. Differential prediction of first clinical response to serotonergic and noradrenergic antidepressants using the loudness dependence of auditory evoked potentials in patients with major depressive disorder. J Clin Psychiatry 2007;68:1206-1212.

16. Park YM, Kim DW, Kim S, Im CH, Lee SH. The loudness dependence of the auditory evoked potential (LDAEP) as a predictor of the response to escitalopram in patients with generalized anxiety disorder. Psychopharmacology (Berl) 2011;213:625-632.

17. Juckel G, Mavrogiorgou P, Bredemeier S, Gallinat J, Frodl T, Schulz C, et al. Loudness dependence of primary auditory-cortex-evoked activity as predictor of therapeutic outcome to prophylactic lithium treatment in affective disorders--a retrospective study. Pharmacopsychiatry 2004; 37:46-51.

18. Baron M, Gershon ES, Rudy V, Jonas WZ, Buchsbaum M. Lithium carbonate response in depression. Prediction by unipolar/bipolar illness, average-evoked response, catechol-O-methyl transferase, and family history. Arch Gen Psychiatry 1975;32:1107-1111.

19. Buchsbaum M, Goodwin F, Murphy D, Borge G. AER in affective disorders. Am J Psychiatry 1971;128:19-25.

20. McKnew DH, Cytryn L, Buchsbaum MS, Hamovit J, Lamour M, Rapoport JL, et al. Lithium in children of lithium-responding parents. Psychiatry Res 1981;4:171-180.

21. Hegerl U, Wulff H, Muller-Oerlinghausen B. Intensity dependence of auditory evoked potentials and clinical response to prophylactic lithium medication: a replication study. Psychiatry Res 1992;44:181-190.

22. Hegerl U, Ulrich G, Muller-Oerlinghausen B. Auditory evoked potentials and response to lithium prophylaxis. Pharmacopsychiatry 1987; 20:213-216

23. Lee SH, Kim JH, Lee JH, Kim S, Park YM, Bae SM, et al. Aberrant response of selective serotonin reuptake inhibitor in two patients with high N100 amplitude slope. Korean J Psychopharmacol 2008;19:341347.

24. Gallinat J, Bottlender R, Juckel G, Munke-Puchner A, Stotz G, Kuss HJ, et al. The loudness dependency of the auditory evoked N1/P2-component as a predictor of the acute SSRI response in depression. Psychopharmacology (Berl) 2000;148:404-411.

25. Park YM, Lee SH, Park EJ. Usefulness of LDAEP to predict tolerability to SSRIs in major depressive disorder: a case report. Psychiatry Investig 2012;9:80-82. 